lees morement there than in the opposite side. There was good breathing all over the right lung, and the percussionsound was normal. Fine crepitation was beard at the back of the left lung, extending as high as the spine of the scapula, and also in the axilla; elsewhere, the breathing was good. There was dnlness on percussion corresponding with the extent over which crepitation was heard. The sputum was thick, tenacious, somewhat rust-coloured, and scanty. There was but little general distress.

He was ordered to take one-eighth of a grain of antimony, with liquor ammonice acetatis mixture, every three hours; and to have low diet and beef-tea.

March 16th. The expectoration was still rust-coloured and scanty. He had slept better. He complained of a dull aching pain. Pulse 80. The auscultatory signs were the same. A blister was ordered to be applied over the back of the left lung.

March 18th. He expressed himself as much better; the pulse had fallen to 68 ; the sputum was less rusty; the crepitation was of a coarser character, and the dulness less. He was put on ordinary diet.

March 19th. Crepitation could only be heard after deep inspiration.

March 21st. The crepitation had disappeared, and the normal lung-sound had returned. The treatment was stopped.

March 23rd. He was discharged.

Case ni. Pleuro.pneumonia, vith Effusion into the Pleural Cavity: Local Bloodletting, etc.: Recovery. Edward Whiteland, aged 19, sailor, moderately stout, and of fair muscular development, was admitted into the hospital April 23, 1860. He was quite well, and had done his work on board ship up to three days before admission. On the night of the 20th April he slept in a draught, and on the following day he began to feel ill, and was obliged to take to his bed in the evening. He had had cough, severe pain in the right side, difficulty of breathing, and rigors.

Seen on the 24th. His face was flushed; the expression Was anxious; pulse 106, rather full and hard ; respirations 40 ; the skin was dry, not very hot; the tongue furred. He complained of severe pain in the right side, luch aggravated by a deep inspiration; there was a short, dry, hacking congh. He could speak only in a whisper.

There was dulness over the lower part of the right side of the chest, extending nearly to the lower angle of the scapula. At the lowest part of the chest the respiratory murmur was absent, but higher up a faint sound was heard, increasing in intensity towards the upper part; it was rapid and catching. No friction sound could be heard; there was exaggerated breathing all over the left lung; the respiration was superior thoracic, the lower intercostals of the right side not acting at all.

He was ordered twelve leeches to the right side; three grains of calomel and a senna draught; and low diet.

April 25th. He had slept a little, and had less pain. Pulse 100 , full ; respirations 34 . He was still much distressed. He was ordered an eighth of a grain of antimony with eight grains of nitrate of potash in acetate of ammonia mixture every four hours; and a pill of three grains of calomel and one of opium at night.

April 26th. He had slept; pulse 104: respirations 26 ; the face was less flushed; the tongue moist. He had less pain in the side, but more in front of the chest; the dulness extended nearly to the spine of the scapula, and there was absence of respiratory murmur nearly to the same level. Eight leeches were ordered to be applied to the right side; the pill to be repeated.

April 27th. He took two grains of calomel and a grain and a half of opium at night

April 28th. He complained of very little pain; he was much relieved by the leeches, which had bled very freely. Pulse 96 ; respirations 30 ; tongue cleaner. He had taken but little food. He was ordered a grain and a half of opium every night, and to continue the mixture without the antimony.

May 2nd. His face had assumed a natural colour and appearance; he was free from pain; the tongue was clean; pulse 110, compressible. He had slept well, and had taken more food, beef-tea, etc. The dulness on the right side extended nearly up to the spine of the scapula, but was less marked; the breath sounds were heard at the upper part, but not below; no friction sound could be detected. A large blister was ordered, and four ounces of wine daily.

May 5th. The pulse was 104; the cough had been very troublesome; he had expectorated a large quantity of tenacious mucus; the reopiration was more eny, and ho was free trom pain. He ras ordered six onnces of wine daily.

May 8th. The pulse was 100; the dulweas on the right side had diminished, but the respiratory sounds could not be beand at the lower part of the lung; he could still speak only in whisper. Chop diet was ordered.

May j1th. There was slight return of fever, and bronchitio rales ware heard at the upper part of the right lung. The nitre mirture was stopped, and a saline, with ipecacuan wine and compound tincture of camphor, was ordered; and a mustard poultice to the chest.

May 13th. Ho had expectorated freely, and was much re. lieved.

May 14th. The pulse was 100; the right lung was free from bronchitic râles; pleural friction.sound was heard at the anterior part; there was still dulness behind, and crepitation was distinctly audible over the lower part of the lung (the fluid in the pleural cavity being now absorbed, the lung came into contact with the walls of the chest, and the crepitus redux of the inflamed organ was heard). He was ordered a pint of porter daily, in addition to his wine.

May 17th. A blister was applied to the right side.

May 18th. He was ondered nitro-muriatic acid, with bitter infusion. He continued to improve steadily, the dulness of the lung gradually diminishing, but his voice being very slow in returning; he was allowed any nourishment he could take.

May 25th. He felt so well that he wished to be discharged. Slight dulness still remained at the lower part of the affected lung.

[To be continued.]

\section{Oitringal Communications.}

\section{G ONOR RH OA I A D S Y PHILITIC R H E M A T IS M.}

By Henty Doncalfe, Esq., M.R.C.S., West Bromwich. [Read bcfore the Qucen's College Medico.Chiturgical Society, Birmingham.]

Rheudratrsar, as connected with and arising from venereal disease, was first described by Sir Astley Cooper, but had been recognised by Cline and others before the time of Sir Astley's notice. That cases of acute and chronic rheumatism, acknowledging a gonorrhœal or syphilitic origin, do occur, is a fact now admitted; but neither any pectuliar type nor modification can be detected, to distinguish such cases from those of simple origin.

The painful chronic, as also the acute rheumatic sequelm of venereal disease, occur generally in enfeebled constitutions, either from hereditary taint, or oftener from the constitutional powers being broken down and exhausted by irregularities of living and other excesses. It is a difficult matter at all times to obtain a true history of such lives; and the wan, pale, and dejected appearance, which the countenances of such patients assume, form not uncommonly the only true indices to the origin of the malady; and patients will so emphatically deny ever having had any venereal taint, that more cases of rheumatic complications may occur than are given credit for.

This paper bas no pretence to research or originality. I have selected a few of the less donbtful cases, to illustrate the forms which the disease may assume; and I shall relate one case to shew that the diagnosis may sometimes be difficult, and that the primary cause may be confounded or mistaken; and I? shall hope to elicit some information on the value of remedies which my own limited experience will oblige me to mention with a certain reserve.

Thus I want to shew-

That rheumatism, when it occurs during the progress of $\mathbb{Q}$ venereal disease, is to be considered not simply as a concomi- 0 tant, but as dependent on that diserse;

That it is important to determine the relative connexion, in order to alleviate the extreme suffering, and prevent the untoward and fearful results which would otherwise ensue.

I wish also to inquire whether there is any form of treat- $F$ ment which is capable of producing a more decided effect in cases of specific than of non-specific origin.

CASE 1. A youth, 10 years of age, had contracted a gonorrhcea, for which he applied to a chemist, and obtained a copaiba mixture, which rapidly chocked the discharge; but 
thin s week he whe seised with severe pain in the right troin. He wo nable to stir in bed whon I first sen him. Fis conntences was expressive of suffering; his stin was hot and perspiring; and his tongus deeply coated, with red edges. There was swelling and tenderness over the anterior and inner apeot of the thigh. The pain was increased by every movement of the limb, and by the merest touch; and towards night it became agoniaing. The urine was scanty and deep coloured, depositing lithates on cooling; the bowels were costive; and there remained a gleety discharge from the urethrs. He had chordee at times, but not extreme; and slight scalding at the time of passing water. He was treated with alkalies, salines, and diaphoretics, without making any progress; and in turn the right knee became affected, then the ankle, and afterwards the left knee. After about eighteen or twenty days, the pain and swelling subsided in the bip; but the knee remained very painful and swollen. It was finally opened, allowing the exit of a large quantity of bloody pus. During the course of the disease, when several remedies had been tried without his having experiencod any relief, colchicum in full doses was combined with the saline mixture; and he said he found more relief from it than from anything else he had taken. In time, the pain and swelling subsided in the left knee and right ankle; his leg was kept on a straight splint; and he was well supported; so that, under chops, porter, steel, and quinine, be contrived to escape with a stiffened bnee.

CASE II. A strumous looking man applied for relief of pain in bis knee. He had some difficulty in walking; but there was no external swelling or redness. On the day following his application at my surgery, I was sent for to see him at his lodgings. I then found an evident fulness about the kneejoint, and pain on handling tho limb, more especially when striking the bottom of the foot. In the course of two or three days, both his eyes became inflamed, and gave him very severe pain. There was great intolerance of light, and he dreaded the approach of night. Salines with colchicum were given; and, after three days, his eyes got well. The pain and fulness diminished in the knee; and a gradually increasing pain seized him in the right side, over the region of the liver; he bad frequent romitings; and in two days be was jaundiced. Purgatives and calomel, with Dover's powder, in timo produced bilious evacuations; and he appeared to be progressing favourably, when the gonorrhwal discharge, which had decreased to a mere globule, and then only on pressing the gland, increased to beyond the extent it had ever been; and he had erratic rheumatic pains in different muscular structures. Colchicum gave him no relief at this time. Guaiacum was substituted, with Dover's powder at bedtime; he was pat on good diet; and the mineral tonics wero afterwards exhibited. For a long time he continued to suffer from wandering pains, and occa. sionally inflammation of the conjunctive; and the remnant of his gonorrhœa proved as intractable as his rlseunatism. He became very impatient with the annoyance of the gleet; and I gave him large doses of copaiba, in a cubeb mixture, which soon cleared away his gleet, and his rheumatism also. He has since had gonorrhœa, but without any other complication.

CASE III. A dissipated young woman requested my attendance in her approaching confinement. She told me that she had led a very irregular life, and had been on the town for several years, till within twelve months prior to the date of her application to me, when she got married, and soon afterwards became pregnant. She had contracted gonorrhoe on more than one occasion; and sometimes used to take medicine, but without any regularity. When first she experienced pains about her limbs, she thought they were due to having taken cold. She had at that time a vaginal discharge, from which she had suffered for a lengthened period. At the time of my inquiries, she complained of much muscular pain (described by herself as gnawing), more particularly about the flexor muscles of the thighs and calves of the legs. The unceasing aching character of the pain was always augmented in the evening; and she had exhausted every posture and change in bed, in the vain hope of finding some ease. With the limited means at her disposal, she had purchased medicines, such as had been recommended by different chemists; but the pain never left her for any length of time, and always got worse at the catamenial periods. Of late, it had been so tormenting that she had resorted to the practice of eating opium, of which she was taking a pennyworth a day. The piece she showed me was the size of a small nut. I gave her the ethereal tincture of guaiacum, which did her much good; and she improved very much both in condition and appearance from the administra. tion of nitric acid; but, soon after her confinement, I lost sight of har, and am nuable to state what was the further progreas of the osee; from the wretched state, howerer, in which she lived, it is probable that she resorted to her former mode of life, and to opium as her only refuge.

These cases present points of practical importance. Before relating them, I said I had selected "the less donltful"; and I used the expression advisedly, for it may be asked whether symptoms analogons to gonorrhoes can exist as an effect or sequence of simple acute rheumatism? To this question I would reply, that I have known an instance of gout which was attended with a discharge like to that of gonorrhcea; and I adduce the testimony of Sir B. Brodie, who, in his Treatise on Diseases of the Joints, says, "I shall have occasion to notice some very remarkable cases of intlammation of the synovial menibrane, preceded by purulent intlummation of the urethre and purulent ophthalmis, to which the name of gonorrhoeal rheuinatism is commonly applied, though the relationship of the disease to rheumatism extends no further than a partial reseml lance in the symptoms." 'The cases quoted as examples will be found from pages 43 to 50 of the last edition. In justice to the subject, I should quote also this passage from the same anthor: "That though inflammation of synovial mem. branes take place independently of gonorrhøal infection, there seems to be no doubt but that it occurs in most instances as a consequence of gonorrhcea."

Of all rheumatisms, the gonorrhcal is by far the most painful; and whilst I have noticed that it may attack various structures, the synovial membranes and sheaths of tendons are its more ordinary seat. As far as my own experience teaches me, I find the muscular form to be less acute, often chronic, and prone to come on after the gonorrhae has existed for some time; whilst the acute articular rheumatism follows the earlier days. The nature of the disease from its onset differs from most other affections of the joints, by the violence of the pain even before any swelling is manifested, and by the tendency for several joints to become affected at the same time or in rapid succession; and differing in its termination by not leaving the joints permanently impaired. Brodie says "the inflammation is for the most part of that kind which terninates in an effusion of serum, and not of coagulated lymph; but sometimes it is more intense, leaving the synovial membrane thickened, and the joint more or less stiff; and I have known a few cases in which the cartilages become ulcerated, with great pain at the time, anchylosis being the ultimate result." Case I presented one of these unusuol terminations.

With respect to the treatment of rheumatic synovitis, the salines seem to be the most serviceable; and I find the hydrochlorate of ammonia, in doses of from eight to ten gruins, with an equal quantity of nitrate of potash, a very useful combination. I would especially mention colchicum, for I believe this particular form of disease to be especially relieved by its administration; and though I would not place reliance on any one remedy in the treatment of any of the forms of rheumatism, I am nevertheless disposed to regard colchicum in these cases as being next to a specific. As the disease becomes chronic or subacute, colchicum ceases to relieve; and, in the acute form, I think $I$ am right in my conclusion when I say that its value is proportioned to a scanty secretion of urine, of high specific gravity, and loaded with lateritious sediment. In the subacute und chronic forms, and especially in muscular rheumatism, guaiacum succeeds to the extent which colchicum does in the acute synovial form. "It acts", Dr. Seymour says, " not as a stimulant, but as an evacuant, provoking purging, perspiration, and a flow of urine in a very violent manner." Sometimes one, sometimes all these effects, he says, follow the use of the medicines. Fuller speaks highly of the use of guaiacum in some of the chronic forms of the disease; and, in cachectic conditions, I can speak to its curative power when combined, as Brodie recommends, with cinchona. Locally, warm fomentations give much ease; occasionully, leeches may be required, but patients do not bear depletion; and while the swelling and redness secm to indicate very acute action, there are often symptoms which lead to an opposito conclusion.

I mentioned in the last cited case, that the patient had for some time been acccustomed to take large doses of opium. So much lately has been said and written about the curative powers of this drug in rheumatism, that one would have thought it ought to have done some good in this case; but its failure was quite in accordance with my own experience of its non-efficacy in this, as in all other rheumatisms. The relief which follows its administration is only what occurs in any other painful disease; and I think it is about as capable of 
curing rheumatism as it is of cancer, when nsed to alles pain in that affection; nay more, I believe that it often does harm by the excessive diaphoresis which it ocossions.

CASE N. The following case will illustrate two varieties of syphilitic rheumatism-the periosteal and muscular; and will suffice to introduce the remarks I shall have to offer in this division of the subject. My patient was a muscular well constitutioned young man, whose boast it was that he had never been obliged to lie by for a day from any illness; indeed, he seemed to be proof against all diseases but gonorrhoe. Eight years ago, after a suspicious intercourse, he had gonorrbca for the first time, with all its symptoms in a superlative degreeswelling of the penis, copious discharge, chordee, buborequiring him to keep his bed for five weeks. Two years afterwards, he agnin had gonorrhoen, but with symptoms 80 modified as to be almost uncared for; and, after consuming some three score capsules, and tanning his urethra with astringent injections, he got wcll, and so remained till within five months before the time when I first saw him. From four to five days after sexual intercourse, he percoived a purulent discharge from the urethra; but, as it was unattended by pain or other annoyance, he made himself contented about it; and it wus about this period, three weeks from the commencement, that with the yeomanry troop he went to do duty for a week prior to the annual review. He was there exposed day after day to a drenching rain; and that, coupled with the evening routine of a soldier's life, no doubt tended to the development of the rheumatism which soon followed. As soon as he settled down to his ordinary employ as a coutractor, he perceived, after walking along the uneven surface of a line of a railway, that his feet wero very tender, especially the heel, about where the plantar fascia arises. He attributed this soreness to his heavy boots, and the uncven surfaces he trod upon; but, as the tenderness increased, and progression was retarded, he consulted a surgeon, who pronounced the disease to be gonorrhueal rheumatism, and recommended him to take iodide of potassium with cinchona, and afterwards tonics with the alkaline carbonates, and an occasional alterative pill. $\mathrm{He}$ persevered with these means for some weeks; but, continuing to get worse, he determined to give up his business, and placed himself under my care.

I first saw him in July 1856. He was confined to his bed, and called my attention particularly to the state of his feet, the right one more especially; and he casually told me he had had a clap, and that there still remained a little discharge. I carefully examined the penis. There was no external swelling, no enlargement or thickening which would mark the site of an internal chancre, nor, as far as I could stretch the lips of the urethra, was there nny sore visible. Ho had not had sore. throat nor enlargement of the inguinal glands, nor any external secondary symptoms. I came to the conclusion that he had chronic urethritis, the result of a gonorrbœa, and rbeumatism of the deep fascia of the feet in connection with that disease. About two montlus from the beginning, he noticed the dis. charge was rather bloody, and remembers to have seen the recurrence of bloody discharge after using an astringent injec. tion. I gave him Plummer's pill every night, and iodide of potassium. He continued the use of the iodide, cinchona being added, till the last week in August, when, finding no im. provement, and some impatience on his part, and some anxiety on the part of his friends, I advised him to see a consulting surgeon, and, if it should be agreed upon, that he should have a change to some sea.coast. His friends overruled him, and he saw a physician, who prescribed quinine, and he went to the Isle of Man. In three weeks, he returned, much the same as when he left, but in addition he noticed that the heel-bones were larger than they should bo; he again saw the physician who had previously preseribed for him, and he was ordered to have the heels painted with iodine, and to take iodide of potassium. At length he consulted a surgeon, who judged from the history, the treatment, and the state of the feet that the disease was probably syphilitic and not gonorrhœal. $\mathrm{He}$ was brought under the influence of mercury; blisters were ap. plied alternately to the sides of the feet, and dressed with blue ointment. A mild caustic injection was used, and the iodide was given when the mercury was discontinued. He soon began to get better; tho swelling of the heels gradually diminished, the pain in the soles of the feet subsided, and he said he felt to have more spring from his feet when he tried to walk. The soles remained very tender for some time, and he had chronic rheunatic pains in his shoulders and about the back and legs. He finally cleared off these pains by recourse to, and continuance with, nitric acid and bark. This no doubt was ase of eyphilis, which I must
candidly confess I had regarded as one of gonorrhoca. The enlargement of the bones would, you might say, have been sufficient indication; but this condition showed itself only after the disease had existed for. some time, and, had it been present at the beginning, it does not neces. sarily follow that the disease must have had its origin in syphilis. This condition is not even confined to cachectic states of the system, though, true, it is usually met with in such persons; and Fuller says, "a cachectic state does not seem to be a condition essential to its development." After the subsidence of the disease in the periosteal covering, the patient had general chronic rheumatism, and this reminds me to remark that when a chronic rheumatic case is presented to $\vec{\nabla}$ us, there are two diseases which it is worth while always to dispose of by inquiry into the history, and by examination of the urine, viz., constitutional syphilis on the one haud, and granular degeneration of the kidney on the other-two very common causes of the disease.

Though iodide of potassium was given in full doses, and $\vec{\omega}$ continued over a lengthened period, there was no amendment; but directly the system was brought under mercury, the iodide 3 became very valuable, and did very much towards facilitating is the cure. Secondary symptoms were delayed, and he had $f$ never had mercury given to salivation; but, in other cases, I .have had some remarkable instances where patients have been $\overrightarrow{0}$ salivated, and afterwards tertiary symptoms (the lepra and psoriasis particularly) and rheumatism have succumbed to the iodide, only after the readministration of mercury.

When the secondary symptoms occur within six or eight $ᄋ$ weeks, the eruption is very commonly papular, and with this $\bullet$ iritis is peculiarly associated, and synovial rheumatism occa. sionally accompanies syphilitic lichen. I am inclined to regard $\subsetneq$ syphilitic iritis as rhenmatism. The iris being a muscular $\mathbb{D}$ structure, covered with a serous membrane, is as liable to $\vec{\infty}$ become affected as the synovial membranes of the joints, which, as Brodie says, very much resemble the peritoneum. The most efficacious treatment is to get the patient under mercury as speedily as possible, to keep up gentle salivation for some time, and afterwards to administer iodide of potas. sium. The joints ofter remain chronically affected, and require liniments, blisters, and other forms of local treatment. Dono-응 van's solution, the bichloride, nitric acid, and sarsaparilla may $\varrho$ also be tried in succession; indeed, in these chronic cases, it $\vec{\overrightarrow{ }}$ is advisable and advantageous to change and vary the remedies 3 repeatedly.

\section{OBSERVATIONS ON THE TREATMENT OF} ASTHMA.

By T. L. Prinhar, Esq., Surgeon, Bideford, North Devon. $\frac{3}{3}$

Mrch has been written of late on the symptoms and treatmento of asthma-perhaps the most painful and apparently alarming disease to which human flesh is heir.

The observations which I have to make on the disease are based on the experience of nearly one hundred cases whicho have come under my medical care. I may not throw much now light on the subject; yet I am desirous to record the result of my experience and my treatment, as well as myñ observations on the peculiarities of this most distressing com plaint, in the hope that some little may be added to thew stock of information which has already been collected on the subject.

It is now many years since a deep rooted impression was made on my mind, by that memorable man, Abernethy, " thate in order to treat disease, whether of a local or constitutiona character, it was absolutely necessary to keep the stomach in that state, so that the fond which was taken into it should bed properly digested, in order to secure good blood, and conse quently healthy secretions and bealthy action of the bods generally; and that in proportion as the surgeon or the phyo sician attended to these particulars, so in proportion would the means placed within the reach of the practitioner be mores or less successful".

With these impressions, which I believe to be most usefub to the medical man, I started in my profession. I had not been long in practice when a severe case of what was called humid asthma presented itself for my treatment. I found that it had been of many years standing, and frequently seemed, from its violence, to threaten the life of the patient. The attack, however, would subside after a copious expectoration 